\title{
IDENTIFICAÇÃO DE PIGMENTOS NATURAIS DE ESPÉCIES VEGETAIS UTILIZANDO-SE CROMATOGRAFIA EM PAPEL
}

\author{
Fabiano Okumura, Márlon Herbert Flora Barbosa Soares e Éder Tadeu Gomes Cavalheiro* \\ Departamento de Química, Universidade Federal de São Carlos, CP 676, 13565-905 São Carlos - SP
}

Recebido em 14/5/01; aceito em 28/11/01

\begin{abstract}
IDENTIFICATION OF NATURAL PIGMENTS FROM VEGETAL SPECIES USING PAPER CHROMATOGRAPHY. The use of natural dyes to demonstrate principles of paper chromatography is proposed. Extraction of the coloring compounds were performed in order to obtain the aglycone form of the anthocyanins present in the crude extracts. Separations were carried out on chromatographic paper with BAW (butanol/acetic acid/water) as mobile phase and the results compared with literature data. The crude extracts were obtained from Tibouchina granulosa, Rododhendron simsii, Impatiens walleriana flowers which are wildely found in Brazil and Phaseolus vulgaris L. grains skin which is the principal ingredient of the world famous "feijoada". Such species were chosen in order to attract the students attention since they are present in their quotidian, in agreement with the new proposals for Brazilian education.
\end{abstract}

Keywords: paper chromatography teaching; anthocyanins; chemical education.

\section{INTRODUÇÃO}

Métodos de separação são um capítulo importante em química, uma vez que, muitas vezes, é necessário isolar substâncias provenientes de um mesmo meio reacional para sua purificação ou separar interferentes para sua adequada identificação e/ou quantificação.

Dentre os diferentes métodos de separação propostos destacamse as técnicas cromatográficas, que constituem um conjunto de procedimentos que vão desde simples técnicas de bancada até sofisticadas metodologias instrumentais ${ }^{1}$.

O ensino dos princípios de tais técnicas pode ser feito usando-se várias fases estacionárias (papéis de filtro ${ }^{2}$, giz $^{3}$, areia ${ }^{4}$, etc.) e diversas amostras, tais como: pigmentos de tecidos vegetais ${ }^{5}$, extratos de frutas $^{6}$, pigmentos naturais $^{7}$, tecidos vegetais clorofilados ${ }^{8}$.

Dentre as várias técnicas cromatográficas, aquela com maior potencialidade didática em cursos básicos de química é a cromatografia em papel, devido à sua simplicidade, facilidade de execução e possibilidade de uso de amostras coloridas, em pequenas quantidades. Segundo Braga ${ }^{9}$, a cromatografia em papel, desenvolvida por Consden, Gordon e Martin ${ }^{10}$, apresenta boa capacidade de resolução e aplica-se principalmente na separação e identificação de compostos polares.

Nosso grupo tem se preocupado em propor novas atividades nas quais são empregados extratos brutos de espécies vegetais contendo antocianinas $^{11-14}$. Tais pigmentos atraem de maneira muito positiva a atenção dos alunos, contribuindo de maneira significativa no processo ensino/aprendizagem de conceitos básicos de química, favorecendo as associações interdisciplinares e usando materiais presentes no cotidiano dos alunos, despertando sua expectativa quanto aos resultados das atividades propostas.

As antocianinas são definidas por Timberlake e Bridle ${ }^{15}$ como derivados de sais flavílicos, solúveis em água, os quais são responsáveis pelas cores atrativas de flores, frutos, folhas, sucos de frutas e até mesmo do vinho. Na natureza, encontram-se associadas a moléculas de açúcares; quando livres destes açúcares são denominadas

\footnotetext{
*e-mail: cavalheiro@dq.ufscar.br
}

antocianidinas (agliconas). A estrutura genérica para uma antocianidina natural é apresentada na Figura 1. Dependendo dos substituintes nas posições R e R' define-se uma antocianidina diferente. Uma descrição das principais antocianidinas encontradas na natureza é apresentada na Tabela 1 .<smiles>[R]c1cc(-c2[o+]c3cc(O)cc(O)c3cc2O)cc([R])c1O</smiles>

Figura 1. Fórmula estrutural de uma antocianidina genérica

Tabela 1. Substituintes R e R' para antocianidinas naturais

\begin{tabular}{ccc}
\hline Antocianidina $^{(a)}$ & $\mathbf{R}$ & $\mathbf{R}$ \\
\hline cianidina & $\mathrm{OH}$ & $\mathrm{H}$ \\
delfinidina & $\mathrm{OH}$ & $\mathrm{OH}$ \\
malvidina & $\mathrm{OCH}_{3}$ & $\mathrm{OCH}_{3}$ \\
pelargonidina & $\mathrm{H}$ & $\mathrm{H}$ \\
peonidina & $\mathrm{OCH}_{3}$ & $\mathrm{H}$ \\
petunidina & $\mathrm{OCH}_{3}$ & $\mathrm{OH}$ \\
\hline
\end{tabular}

(a) As estruturas correspondentes são relacionadas com a fórmula estrutural genérica apresentada na Figura 1

Harborne ${ }^{16}$ propôs um método simples para separar e identificar antocianidinas presentes em extratos vegetais brutos, usando cromatografia em papel. O método foi aplicado por Curtright et al. ${ }^{17}$ na identificação de pigmentos de frutas e de repolho roxo em um experimento didático.

Considerando-se as vantagens do uso de pigmentos naturais descritos anteriormente ${ }^{11-14}$, desenvolveu-se um procedimento para a de- 
monstração de princípios básicos de cromatografia em papel, na separação e identificação de antocianidinas presentes em extratos brutos de quaresmeira (Tibouchina granulosa), beijinho (Impatiens waleriana), azaléia (Rododhendron simsi) e do feijão preto (Phaseolus vulgaris, FP).

\section{MATERIAIS E MÉTODOS}

\section{Reagentes e soluções}

Foram utilizados reagentes de grau analítico, PA, sem purificação prévia. As soluções foram preparadas com água destilada. Assim, o procedimento é simplificado, não havendo necessidade de se utilizar técnicas e materiais sofisticados, o que facilita a aplicação didática desta atividade experimental.

\section{Obtenção dos Extratos}

Os extratos das flores podem ser preparados segundo o procedimento descrito por Harborne ${ }^{16}$, que consiste na extração de pigmentos a partir de pequenas quantidades do tecido vegetal, por imersão em cerca de $5 \mathrm{~mL}$ de solução etanólica de $\mathrm{HCl}$ a $1 \%$, em um tubo de ensaio mantido a $80{ }^{\circ} \mathrm{C}$ por cerca de $40 \mathrm{~min}$. O extrato obtido é suficientemente concentrado e pode ser utilizado diretamente, sem evaporar. No entanto, cabe salientar que este é um procedimento específico para pequenas quantidades de tecidos vegetais, o qual permite obterem-se as antocianinas na forma livre de açúcares, as antocianidinas.

Alternativamente, pode-se usar o procedimento descrito por Couto et al. ${ }^{11}$ e Soares et $a l .{ }^{12}$ que permite a obtenção de maiores quantidades de extrato. Neste caso, $300 \mathrm{~g}$ do tecido vegetal são imersos em $300 \mathrm{~mL}$ de etanol por $48 \mathrm{~h}$ e, posteriormente, o solvente é eliminado em evaporador rotatório sob vácuo.

Para o FP é necessário um aquecimento brando $\left(10 \mathrm{~min} \mathrm{a} 60^{\circ} \mathrm{C}\right)$, com água suficiente para cobrir os grãos. A seguir, adiciona-se o dobro de água e aguarda-se 10 min para a extração completa dos pigmentos, filtra-se e elimina-se o solvente por evaporação sob vácuo.

Em todos os casos, o processo de evaporação sob vácuo leva à obtenção de antocianidinas (agliconas), segundo Harborne ${ }^{16}$.

\section{Papel utilizado como suporte}

Utilizou-se tanto o papel de filtro qualitativo como o papel cromatográfico WHATMAN número 3, para comparação.

\section{Fase móvel}

Como fase móvel foi utilizado o eluente conhecido como BAW, uma solução de butanol, ácido acético e água na proporção 4:1:5 $(\mathrm{v} / \mathrm{v})^{16}$.

\section{Procedimentos}

As amostras foram preparadas segundo o método de Harborne ${ }^{16}$ e foram obtidos cromatogramas nos dois tipos de papel, de acordo com o procedimento descrito a seguir.

Usando-se um conta-gotas, aplicou-se a amostra a cerca de 1,5 $\mathrm{cm}$ de uma das extremidades de um pedaço de papel com aproximadamente $5 \mathrm{~cm}$ de largura por $15 \mathrm{~cm}$ de altura. Em um béquer com capacidade para $1000 \mathrm{~mL}$ foram colocados aproximadamente $50 \mathrm{~mL}$ da fase móvel, na qual mergulhou-se o papel até cerca de $0,3-0,5 \mathrm{~cm}$ da extremidade próxima à amostra. $\mathrm{O}$ papel contendo a amostra foi preso na parte superior do béquer usando-se um lápis. Como alternativa, pode-se utilizar um pedaço de arame. O sistema foi tapado com filme de PVC de cozinha ou um vidro de relógio, aguardandose até que a fase móvel percorresse $10 \mathrm{~cm}$ do papel.

A fase móvel leva cerca de 2 a 3 horas para percorrer os $10 \mathrm{~cm}$ estabelecidos. Após este tempo, o papel foi secado com um secador de cabelos e revelado sob luz ultra-violeta $(\lambda=365 \mathrm{~nm})$. Também foi usado iodo como revelador, entretanto não foram obtidos resultados satisfatórios. A partir do centro da mancha revelada, mediu-se a distância percorrida pela amostra (em centímetros) e dividiu-se pela distância estabelecida de $10 \mathrm{~cm}$, determinando-se o Rf experimental, de acordo com a Equação 1:

$R f=(d / 10) \times 100$

na qual d é a distância percorrida pelo centro da mancha, em centímetros.

A identificação dos pigmentos foi feita comparando-se os valores de Rf determinados para cada mancha com os valores de Rf descritos na literatura ${ }^{16}$, para as antocianidinas mais comuns.

A Figura 2 apresenta o esquema geral utilizado para os experimentos cromatográficos com os extratos de quaresmeira, azaléia, beijinho e FP, utilizando-se BAW como fase móvel.

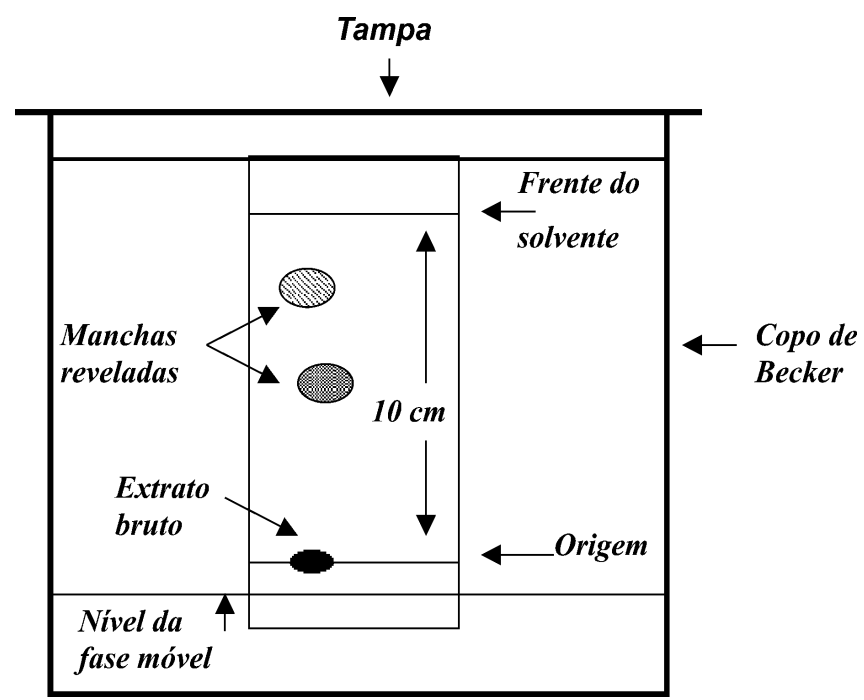

Figura 2. Esquema geral utilizado para os experimentos cromatográficos com os extratos de FP, azaléia, beijinho e quaresmeira, utilizando como fase móvel, BAW

\section{RESULTADOS E DISCUSSÃO}

Um cuidado deve ser tomado quando do procedimento de extração para que se tenha certeza de obter antocianinas ou antocianidinas (agliconas). Isso se deve à facilidade de hidrólise dos açúcares presentes, quando a extração é feita em meio ácido sob aquecimento.

Segundo Harborne ${ }^{16}$, a hidrólise completa dos açúcares ligados à antocianidina central ocorre em $1 \mathrm{~h} \mathrm{a} 60{ }^{\circ} \mathrm{C}$, na presença de $\mathrm{HCl} 1 \%$ em etanol. Em tempos intermediários, pode ocorrer a hidrólise parcial obtendo-se misturas de agliconas e compostos ligados a açúcares.

Harborne também afirma que a hidrólise completa é obtida quando o extrato etanólico é submetido à roto-evaporação, sob aquecimento, para eliminação do solvente. Para se obter as antocianinas, a extração deve ser feita com metanol sem ácido e sem aquecimento.

No presente trabalho, os procedimentos de extração e secagem dos extratos brutos levam à obtenção de antocianidinas (agliconas). 
A Tabela 2 apresenta as principais antocianidinas e os seus respectivos valores de $\mathrm{R}_{\mathrm{f}}$ descritos por Harborne ${ }^{16}$, para condições experimentais semelhantes às utilizadas em nosso trabalho.

Os resultados obtidos para todos os extratos são apresentados na Tabela 3, a qual apresenta valores de Rf para extratos preparados segundo Harborne ${ }^{16}$ e trabalhos anteriores desenvolvidos pelo grupo $^{11,12}$. Os resultados descritos na Tabela 3 são concordantes para os dois procedimentos de extração adotados e levaram à identificação das antocianidinas presentes por comparação dos valores de Rf da Tabela 2 .

Para o extrato bruto de FP, obtiveram-se 2 manchas cromatográficas atribuídas à presença de delfinidina e pelargonidina, de acordo com as Tabelas 2 e 3.

Os resultados obtidos neste trabalho para o extrato de FP, considerando-se os valores de Rf da Tabela 2, são coerentes com o trabalho de Takeoka et al..$^{18}$ quanto à identificação de delfinidina no extrato de FP. No entanto, embora aqueles autores tenham encontrado petunidina ao invés de pelargonidina, descrevem outros trabalhos, anteriores ao deles, nos quais se encontram pelargonidina ou cianidina dependendo sempre da região geográfica de procedência do extrato.

Para o extrato de azaléia, os resultados também são descritos na Tabela 3, sendo confirmadas as presenças da petunidina e da malvidina, cujo valor de $\mathrm{R}_{\mathrm{f}}$ observado concorda com o descrito na literatura ${ }^{16}$. Já De Loose ${ }^{19}$ além destes, descreve a presença de cianidina, a qual não foi detectada neste experimento. A ausência deste pigmento pode estar associada à hidrólise incompleta da cianina original (glicosilada), cujo valor de $\mathrm{R}_{\mathrm{f}}$ é sistematicamente menor ou à diferença na região geográfica de coleta, segundo o próprio autor.

Tabela 2. Valores de literatura ${ }^{16}$ para os $\mathrm{Rf}$ das principais antocianidinas, obtidos de cromatografia em papel

\begin{tabular}{lc}
\hline Pigmento & Rf $^{\mathbf{a}}$ \\
\hline pelargonidina $(\mathrm{Pl})$ & 80 \\
cianidina $(\mathrm{Cy})$ & 68 \\
peonidina $(\mathrm{Pe})$ & 71 \\
delfinidina (Df) & 42 \\
petunidina (Pt) & 52 \\
malvidina (Mv) & 58 \\
\hline
\end{tabular}

${ }^{\mathrm{a}} R_{f}=(d / 10) \times 100$, em que $\mathrm{d}=$ distância percorrida pela mancha (cm), usando o eluente BAW (butanol/ácido acético/água 4:1:5 v/v) em papel cromatográfico.
Para o extrato de quaresmeira, os resultados descritos na Tabela 3 permitem a identificação da petunidina e da pelargonidina enquanto para o beijinho, encontrou-se delfinidina e peonidina.

Em relação aos resultados obtidos para a quaresmeira e para o beijinho, os valores de Rf foram caracterizados segundo os valorespadrão constantes da Tabela 2. No entanto, não foram encontrados na literatura trabalhos que descrevam antocianinas (antocianidinas) presentes nas flores destas espécies.

A fase móvel que apresentou os melhores resultados foi o BAW. Entretanto, embora também tenham sido realizados alguns testes cromatográficos usando amônia e forestal ( $\mathrm{HCl}$, ácido acético e água), como fase móvel, os resultados obtidos não foram satisfatórios quanto à resolução das manchas.

Utilizou-se também papel de filtro como alternativa ao papel cromatográfico, com o objetivo de reduzir custos e tornar o experimento mais fácil de ser executado em escolas. Os resultados foram similares aos obtidos com papel cromatográfico, o que confirma a possibilidade de realização em práticas laboratoriais com custo inferior.

Seria desejável a comparação dos resultados com os $\mathrm{R}_{\mathrm{f}}$ de padrões para uma maior confiabilidade dos resultados. Ocorre que padrões comerciais de antocianidinas são raros e de alto custo. Assim, uma vez que o procedimento proposto por Harborne ${ }^{16}$ é consagrado como um método para a identificação de antocianidinas de vegetais, os resultados obtidos são considerados confiáveis. O isolamento e a caracterização das antocianidinas através de técnicas como cromatográficas (CG ou CLAE), associadas à espectrometria de massas à espectroscopia na região do infravermelho poderiam fornecer tais padrões. Entretanto, tais metodologias escapam à proposta didática simples que se busca neste trabalho.

\section{AGRADECIMENTOS}

À FAPESP pelo auxílio financeiro (98/13873-5) e bolsa de M. H. F. B. Soares e ao Programa PIBIC CNPq/UFSCar pela bolsa de F. Okumura.

\section{REFERÊNCIAS}

1. Collins, C. H. Em Introdução a Métodos Cromatográficos; Collins, C. H.; Braga, G. L.; Bonato, P. S., eds.; Editora da Unicamp: Campinas, 1997, p. 13.

2. Croft, L.R.; Haghighis, S.; J. Chem. Educ. 1977, 54, 112.

3. Wollrab, A.; J. Chem. Educ. 1975, 52, 809.

4. Lalitha, N.; J. Chem. Educ. 1994, 71, 432.

5. Kimbrough, D. R.; J. Chem. Educ. 1992, 69, 987.

Tabela 3. Valores de Rf para os extratos de FP, Azaléia, Beijinho e Quaresmeira

\begin{tabular}{|c|c|c|c|}
\hline \multirow[b]{2}{*}{ Extrato } & \multicolumn{2}{|c|}{$\mathrm{Rf}^{\mathrm{a}, \mathrm{b}, \mathrm{c}}$ para extração segundo: } & \multirow[b]{2}{*}{ Atribuição } \\
\hline & Harborne $^{16}$ & LATEQS $^{11,12}$ & \\
\hline Feijão Preto & $43,0 \pm 2,5$ & $42,5 \pm 0,7$ & delfinidina (Df) \\
\hline (Phaseolus vulgaris) & $78,0 \pm 1,7$ & $79,5 \pm 1,1$ & pelargonidina $(\mathrm{Pl})$ \\
\hline Azaléia & $48,1 \pm 2,8$ & $50,8 \pm 1,7$ & petunidina $(\mathrm{Pt})$ \\
\hline (Rododhendron Simssi) & $57,2 \pm 1,3$ & $56,3 \pm 0,9$ & malvidina (Mv) \\
\hline Beijinho & $41,0 \pm 0,6$ & $41,5 \pm 0,5$ & delfinidina (Df) \\
\hline (Impatiens walleriana) & $73,0 \pm 1,1$ & $72,5 \pm 0,8$ & peonidina $(\mathrm{Pe})$ \\
\hline Quaresmeira & $50,9 \pm 0,4$ & $51,2 \pm 1,1$ & petunidina $(\mathrm{Pt})$ \\
\hline (Tibouchina granulosa) & $85,3 \pm 2,0$ & $84,1 \pm 1,3$ & pelargonidina $(\mathrm{Pl})$ \\
\hline
\end{tabular}

a - média de três determinações; $b$ - para comparação dos resultados foram obtidos os Rf usando extratos obtidos segundo a metodologia de Harborne $^{16}$ e pelo nosso grupo, para as flores ${ }^{11}$ e para o feijão preto ${ }^{12}$; $\mathrm{c}$ - LATEQS = Laboratório de Análise Térmica, Eletroanalítica e Química de Soluções 
6. Curtright, R.D.; Rynearson, J. A.; Markwell, J.; J. Chem. Educ. 1994, 71, 682.

7. Séquin-Frey, M.; J. Chem. Educ. 1981, 58, 301.

8. Anwar, M. H.; J. Chem. Educ. 1963, 40, 29.

9. Braga, G. L. Em Introdução a Métodos Cromatográficos; Collins, C. H.; Braga, G. L.; Bonato, P. S., eds.; Ed. Unicamp: Campinas, 1997, p. 31.

10. Consden, R.; Gordon, A. H.; Martin, A. J. P.; Biochem. J. 1944, 38, 224.

11. Couto, A.B.; Ramos, L. A.; Cavalheiro, E. T. G.; Quim. Nova 1998, 21, 221

12. Soares, M. H. F. B.; Antunes, P. A.; Cavalheiro, E. T. G.; Quim. Nova 2001, 24,408 .

13. Soares, M.H.F.B.; Boldrin-Silva, M.V.; Cavalheiro, E. T. G.; Eclet. Quim. 2001, 26, 225.
14. Soares, M. H. F. B.; Couto, A. B.; Ramos, L. A.; Cavalheiro, E. T. G.; Book of Abstracts, XI Euroanalysis, Lisboa, Portugal, 2000.

15. Timberlake; C. F.; Bridle, P. Em The Flavonoids - Part I; Harborne, J.B.; Mabry, T. J.; Mabry, H., eds.; Academic Press: New York, 1975, p. 215.

16. Harborne, J.B.; Phytochemical Methods - A Guide to Modern Techniques of Plant Analysis, Chapman and Hall: London, 1973, p. 33.

17. Curtright, R.D.; Rynearson, J. A.; Markwell, J.; J. Chem. Educ. 1996, 73, 306.

18. Takeoka, G. R.; Dao, L. T.; Full, G. H.; Wong, R. Y.; Harden, L. A .; Edwards, R. H.; Berrios, J. de J.; J. Agric. Food. Chem. 1997, 45, 3395.

19. De Loose, R.; Phytochemistry 1969, 8, 253. 